\title{
Hypothesis of Endogenous Anticholinergic Activity in Alzheimer's Disease
}

\author{
Kimiko Konishia, b Koji Horia ${ }^{a}$ Masayuki Tanic Hiroi Tomioka ${ }^{a}$ Yuka Kitajimad $^{d}$ \\ Norihisa Akashi ${ }^{\mathrm{e}}$ Atsuko Inamoto $^{a}$ Kenzo Kurosawa ${ }^{a}$ Haijime Yuda $^{a}$ \\ Takahiro Hanashi ${ }^{a}$ Hiromi Ouchi ${ }^{a}$ Misa Hosoi ${ }^{a}$ Mitsugu Hachisu ${ }^{f}$

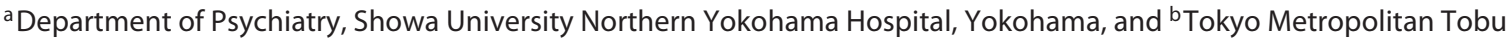 \\ Medical Center for Persons with Developmental/Multiple Disabilities, ' Department of Psychiatry, Showa University East \\ Hospital, 'd Department of Anesthesiology, School of Medicine, Juntendo University, 'Department of Psychiatry, Showa \\ University Karasuyama Hospital, and f Department of Pharmaceutical Therapeutics, Division of Clinical Pharmacy, \\ School of Pharmacy, Showa University, Tokyo, Japan
}

\section{Key Words}

Alzheimer's disease · Anticholinergic activity ·

Acetylcholine $\cdot$ Endogenous appearance $\cdot$ Serum anticholinergic activity

\section{Abstract \\ In this article, we review and repropose our hypothesis of the endogenous appearance of anticholinergic activity (AA) in Alzheimer's disease (AD). First, we introduce our previous ar- ticles and speculate that, because acetylcholine (ACh) regu- lates both cognitive function and inflammation, downregu- lation of this neurotransmitter causes upregulation of the inflammatory system. AA then appears endogenously with the production of cytokines and the downregulation of $A C h$ in $A D$. To support our hypothesis, we present a female $A D$ patient whose $A A$ was considered to occur endogenously through her AD pathology. Her serum anticholinergic activ- ity (SAA) was positive at her first visit to our memory clinic, was negative at the 1-year and 2-year follow-up visits, and had become positive again by 3 years. We speculate that the initial positive SAA was related to her AD pathology plus mental stress, and that her SAA at 3 years was related to her}

(c) 2015 S. Karger AG, Basel

$1660-2854 / 15 / 0153-0149 \$ 39.50 / 0$
AD pathology only. Consequently, we believe that 2 patterns of SAA positivity (and therefore AA) exist. One occurs when the downregulation of $A C h$ reaches a critical level, and the other occurs with the addition of some other factor such as medication, induced illness or mental stress that causes AA to affect $A D$ pathology. Finally, we consider the pharmacotherapy of $A D$ based on the proposed hypothesis and conclude that cholinesterase inhibitors can be used to prevent rapid disease progression, whereas $\mathrm{N}$-methyl-D-aspartate receptor antagonists should be reserved for the treatment of $A D$ that is already in a stage of rapid progression. We also propose a staging schema for patients with AD.

(c) 2015 S. Karger AG, Basel

\section{Introduction}

We have previously proposed a hypothesis of the endogenous appearance of anticholinergic activity (AA) in Alzheimer's disease (AD) [1-3]. To confirm this hypothesis, we needed to observe the presence of serial longterm serum anticholinergic activity (SAA) [4], a peripheral marker of AA [1-3], in patients diagnosed with $\mathrm{AD}$.

\section{KARGER 125}

E-Mail karger@karger.com www.karger.com/ndd
Koji Hori, MD, PhD

Department of Psychiatry, Showa University Northern Yokohama Hospital 35-1 Chigasakichuo, Tsuzukiku

Yokohama City, Kanagawa 224-8503 (Japan)

E-Mail kojihori@med.showa-u.ac.jp 
In this review, we include a reference case in support of our hypothesis. A woman with $\mathrm{AD}$, whose SAA was initially positive at her first visit to our memory clinic, subsequently returned to normal for 3 years and then became positive again as her condition deteriorated.

We first introduce our previous research in support of the endogenous appearance of AA in AD [1-3]. Secondly, we present the case report in further detail in light of the possibility of endogenous AA. Thirdly, we speculate that the clinical course and stage of AD are consistent with the proposed hypothesis. Fourthly, we consider the pharmacotherapy of $\mathrm{AD}$ based on the hypothesis of an endogenous appearance of AA in AD. Finally, we show the points which need special attention in the pharmacotherapy for AD patients.

\section{Introduction of Endogenous Emergence of AA in AD}

We previously evaluated and reported the relationship between AA, demographic and clinical features: sex, educational level, age at dementia onset and age at the time of test; severity of dementia, cognitive function and behavioral and psychological symptoms, and the number of prescribed psychotropic medicines. This was tested among $76 \mathrm{AD}$ patients treated at the National Shimofusa Hospital (Chiba, Japan) from May 1, 2003, to March 31, 2005. We evaluated AA using SAA, which is a peripheral marker considered to reflect the AA burden in the central nervous system $[5,6]$. Among the 76 patients with AD, 26 were SAA positive; the mean marker for SAA was $4.14 \pm$ $2.70 \mathrm{nM}$, with significantly lower global cognitive function and severer dementia, delusions, hallucinations and diurnal rhythm disturbances $(\mathrm{p}<0.05)$. Moreover, many more psychotropic medicines were prescribed in the SAA-positive group. Delusions and diurnal rhythm disturbances were positively correlated with SAA in logistic regression analysis, and not only global cognitive function, but also moment memory and short-term memory were significantly lower in the SAA-positive group than in the SAA-negative group $(\mathrm{n}=50)$. These results were consistent with previous works $[7,8]$. Consequently, these findings led to our proposed hypothesis of AA in $\mathrm{AD}$; that is, AA appeared endogenously and, when it did appear, it made the progression of $\mathrm{AD}$ more rapid. We postulated that because psychotropic medicines are often clinically prescribed for the psychiatric symptoms of agitation and psychosis in $\mathrm{AD}[9,10]$, there may be cyclic relationships among these factors, i.e. prescription of psychotropic medications, appearance of AA and psychiatric symptoms such as delusion and hallucinations, we termed this the 'vicious cycle of AA in AD' [6]. Because medicines are often prescribed for clinical agitation and psychosis in $\mathrm{AD}$, it is possible that the initial event is the appearance of AA [6]. Therefore, we further speculated that AA not only caused the cognitive deterioration by antagonizing acetylcholine (ACh), but also accelerated the AD pathology by inhibiting muscarinic receptors (i.e. downregulating ACh) [5]. In fact, Perry et al. [11] reported that amyloid plaque densities were more than 2.5 -fold higher in cases treated with long-term antimuscarinic medications (more than 2 years) compared with those with nontreatment or short-term treatment (less than 2 years) with antimuscarinic medications. Lu and Tune [12] have also commented that chronic exposure to anticholinergic medications accelerated the clinical course of AD.

Based on these findings, we sought to examine why AA appeared endogenously in $\mathrm{AD}$ and why it might accelerate $\mathrm{AD}$ pathology [1-3]. In general, AA results primarily from the use of prescription drugs, particularly those with potent AA contents and complex administration regimens [13]. However, Flacker and Lipsitz [14] reported that SAA disappeared without medication changes following the amelioration of acute physical illnesses and commented that SAA may reflect a nonspecific stress response to illness in the elderly. Plaschke et al. [15] also reported that SAA became positive from both extrinsic and intrinsic factors and proposed that mental stress (and raised cortisol) was a causal factor for SAA. These authors concluded that endogenous AA occurred secondary to either illness [14] or stress [15]. Moreover, they commented that intrinsic factors play an equal role, even if AA is induced by prescribed medications. Consequently, the anticholinergic load cannot be inferred by an individual's medication use alone; prescribed medications were not the only reason for AA, suggesting that endogenous mechanisms must therefore exist. Based on the work of Flacker and Lipsitz [14], we reviewed the relationship between ACh and inflammation, and between inflammation and $\mathrm{AA}$ [16]. In $\mathrm{AD}$, we speculated that reduced cholinergic neuronal activity due to degeneration causes AA through inflammatory processes [16]. When cholinergic deficiency reaches a threshold level, anti-inflammatory activity cannot inhibit inflammatory system activation, and AA appears with the release of cytokines $[17,18]$. Therefore, we consider that it is possible that neuronal immunoreactions (inflammation) are induced by the reduction of ACh activity in the brain of patients with $\mathrm{AD}[1-3]$. Hence, we proposed the 'endogenous anticholinergic hypothesis in AD’ (fig. 1; courtesy 
Fig. 1. We speculate that ACh degradation not only causes cognitive dysfunction and behavioral and psychological symptoms of dementia (BPSD), but also induces inflammation in the central and peripheral nervous systems, which induces AA in both systems through cytokine pathways. Moreover, AA increases amyloid pathology and further decreases ACh. This is referred to as the 'endogenous AA cascade'. NMDA = $\mathrm{N}$-methyl-D-aspartate. Image courtesy of Hori et al. [1]. The procedure was permitted by the Japanese Society of Neuropsychopharmacology (Tokyo, Japan).

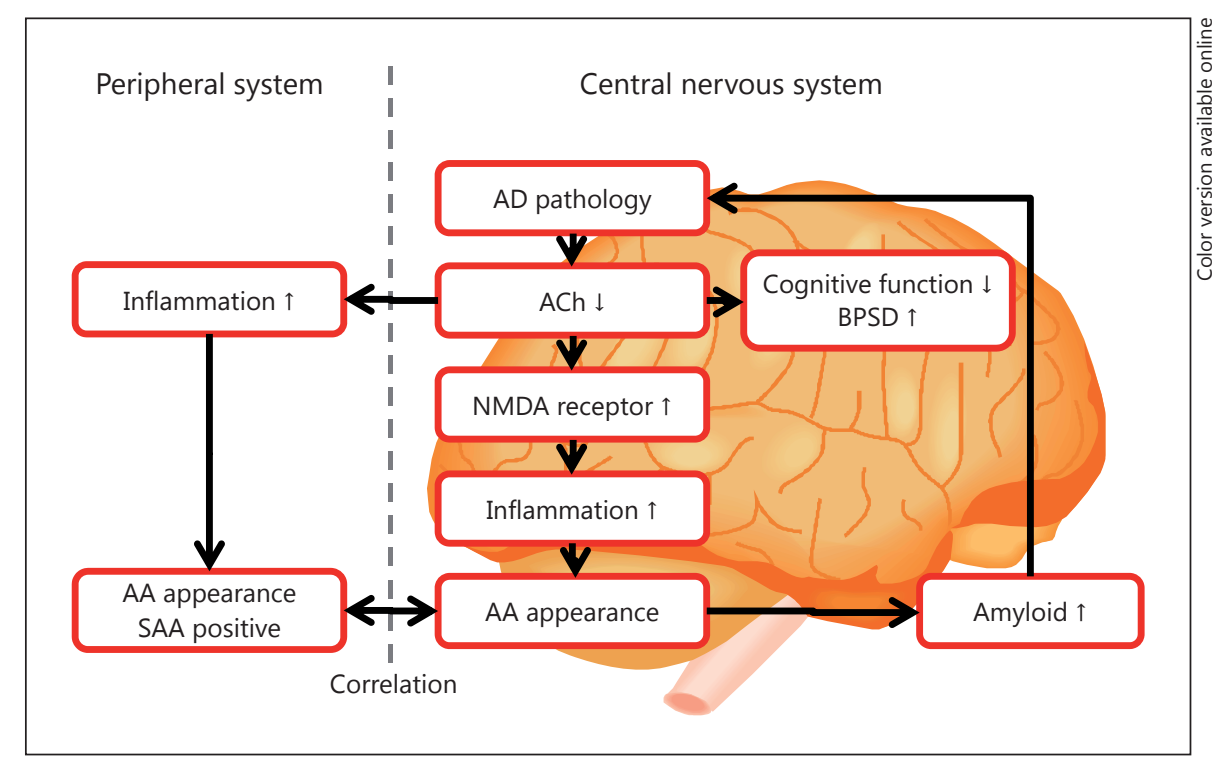

of Hori et al. [1]). Here ACh downregulation causes downregulation of the anti-inflammatory pathway, which in turn causes upregulation of the inflammatory pathway (the cholinergic anti-inflammatory pathway) $[19,20]$ and hyperactivity of inflammation-generated AA via Nmethyl-D-aspartate (NMDA) receptor hyperactivity [21]. Regulation of the NMDA receptors occurs probably via the nicotinic ACh receptors [22]. Because the characteristic feature of $\mathrm{AD}$ is $\mathrm{ACh}$ downregulation [23], when the level of ACh reaches a critical level (i.e. moderately severe disease), AA is endogenously generated, which causes the rapid progression of cognitive decline. We refer to this vicious cycle as the 'endogenous cascade of $\mathrm{AA}$ in $\mathrm{AD}$ ' [1, 2] and refer to this acceleration of $\mathrm{AD}$ as 'self-perpetuating progression of $\mathrm{AD}^{\prime}[1,3]$.

\section{Case Presentation}

To prove our hypothesis, we need long-term follow-up data for patients with stable $\mathrm{AD}$ and medications. We have previously presented the case of a 76-year-old man with moderate AD whose SAA was positive when his memory disturbance, disorientation, apathy and aphasia deteriorated; his SAA resolved after 3 months' treatment with an NMDA receptor antagonist [24]. Nevertheless, this case did not involve long-term follow-up data. Indeed, such long-term follow-up data is needed because, when patients' $\mathrm{AD}$ subsequently progresses rapidly and their SAA becomes positive in the absence of mental stress, physical illness or additional medications, we can provide evidence of the endogenous appearance of AA in AD. This work is currently in progress; however, during this process we encountered an interesting and particularly relevant case. Here, we present the case of a patient with $\mathrm{AD}$ and consider the possibility that her disease course supports the endoge- nous appearance of AA. Both the patient and her husband gave written informed consent for the publication of this case report. Concerning the assessment of SAA, patient information was kept confidential at sample submission, and SAA was assayed according to the receptor binding assay protocol of Tune and Coyle [4] at Mitsubishi Chemical Medience Corp. (Kumamoto, Japan).

A 74-year-old woman presented at the memory clinic of our hospital with memory disturbances for 2 years. She had moved to a new residence 3 months before visiting our clinic, and this shift had caused her excessive stress. Since the move, she had been troubled by visual hallucinations, apathy and irritability. She told us: 'I can see someone ... but he soon disappears, and my husband says there is no one there.' She also complained of blurred vision for 3 months before her first visit to our hospital. On initial examination, her Mini-Mental State Examination (MMSE) [25] score was 26 and her SAA marker concentration was $4.28 \mathrm{nM}$. We diagnosed mild cognitive impairment (MCI) [26] with AD because her cognitive function was relatively spared, although she and her husband had noted memory disturbances. Diagnosis was made using a scale developed by a working group of the National Institute of Neurological and Communicative Disorders and Stroke in collaboration with the Alzheimer's Disease and Related Disorders Association [27]. We prescribed donepezil (5 mg daily), and her visual hallucinations, blurred vision and apathy resolved shortly after treatment had started. Her MMSE score further decreased to 24 after 1 year of treatment, although her SAA was undetectable $(<1.95 \mathrm{nM})$. We have already reported her outcomes over the first year from her first visit to our clinic [28].

At 1 year 4 months after her first presentation, her husband complained that her memory disturbance had worsened. We increased the donepezil dose to $10 \mathrm{mg}$ daily and, although her SAA remained undetectable, her visual hallucinations and memory disturbances did not diminish. She then continued with stable, daily activity, and her MMSE score decreased to 22 by 2 years, still with an undetectable SAA level. By 2 years 5 months after her first visit to our memory clinic, her husband complained that her memory disturbance had again worsened, so we added memantine to her 
Fig. 2. Graphical representation of the clinical course of a 74-year-old woman with AD. SAA was positive at the first visit to our memory clinic and resolved for 3 years, at which point it became positive again. We present her disease course and consider the possibility that it reflects the endogenous appearance of AA with disease progression. $\mathrm{X}=$ Initial examination.

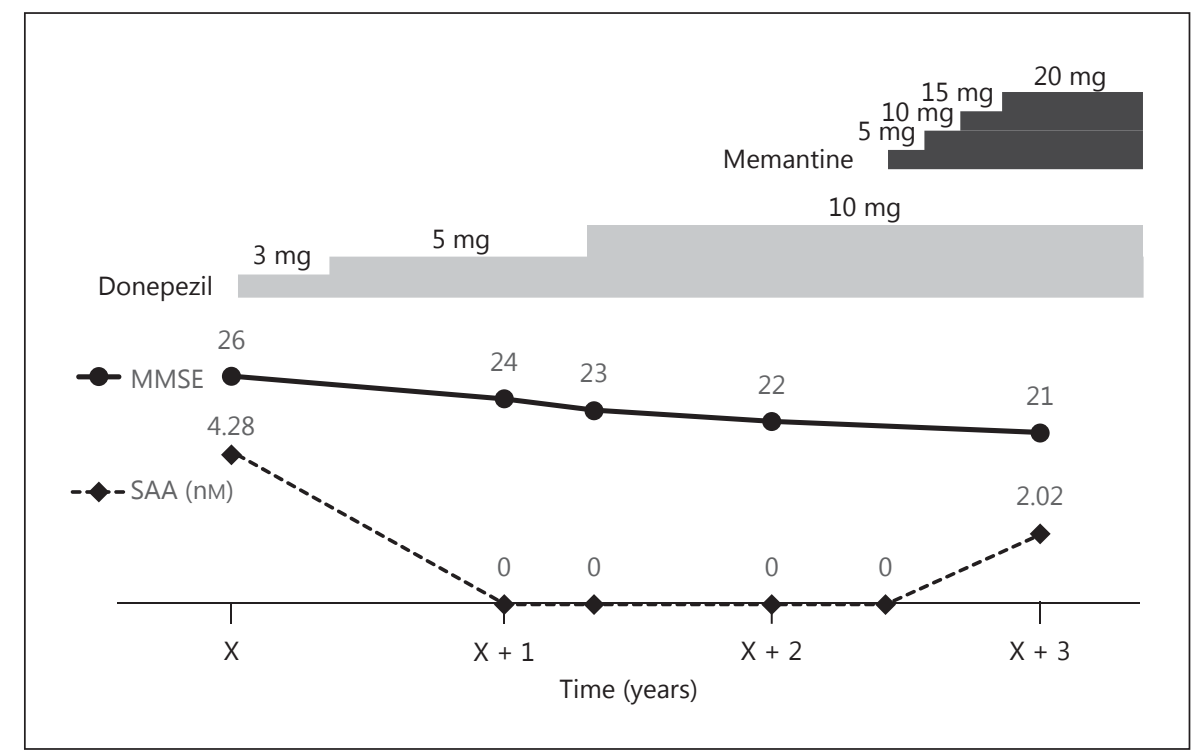

regimen, and her SAA continued to remain undetectable. However, at 3 years her MMSE score decreased to 21 and her SAA became positive $(2.02 \mathrm{nM})$. Moreover, although her clinical symptoms gradually deteriorated until 3 years including her memory disturbances, visual hallucinations and glaucoma were worse after 3 years. From 3 years 10 months, she has been confused with visual hallucinations (fig. 2).

In this patient, we believe that SAA was positive at her first visit because she was burdened by both her AD pathology and stress [28]. Conversely, we speculate that, 3 years later, the increased SAA was due to the fact that ACh downregulation had reached a critical level that allowed AA to appear endogenously. After her first visit, there were no changes in her medical comorbidities, mental stress or anticholinergic medication. However, her eyedrop dose was increased, donepezil was increased and memantine added (after 2 years). We propose that downregulation of ACh caused hyperactivity of the inflammatory system, cytokine release and AA through the inflammatory system [28], with AA causing an amyloidogenic process and further downregulation of ACh [13] (fig. 1). According to this hypothesis, memantine should be able to ameliorate the effects of AA, which is consistent with the earlier case report in which SAA decreased to the point of becoming undetectable after the prescription of memantine [24]. Consequently, we argue that her SAA became positive at 3 years for some reason other than medication, physical illness or mental stress, and we speculate that its appearance was endogenous. That is, the change to SAA positivity occurred when ACh had reached a critical level that allowed the progression of $\mathrm{AD}$ : the 'endogenous anticholinergic cascade' [1-3] had been triggered.

Her SAA marker concentration was elevated at $4.28 \mathrm{nM}$ at her first visit to our clinic and, after a period of decreased levels, became elevated to $2.02 \mathrm{nM}$ at 3 years, which is plausible. We previously reported that SAA caused by medication had $10.8 \mathrm{nM}$ [29] and that SAA caused endogenously had $2.38 \mathrm{~nm}$ [24]. Therefore, SAA caused by $\mathrm{AD}$ plus another factor results in relatively high increases, while SAA caused by AD pathology alone results in relatively low increases. Generally, any SAA level above the quantita- tive marker detection limit ( $\geq 1.95 \mathrm{nM}$ in our study) is considered positive. According to previous studies, SAA has been considered positive when its equivalent was $\geq 1.95 \mathrm{nM}$ and undetectable when $<1.95$ nM. Nevertheless, because a low SAA can cause AA in the central nervous system, we should assume that SAA might also be positive if its marker equivalent is at any level above zero (fig. 3; courtesy of Hori et al. [2]).

To our knowledge, this is perhaps the first case presentation to show a possible endogenous appearance of AA in AD after longterm observation. We are grateful to the patient and her family for their cooperation.

\section{Clinical Applications Based on the Hypothesis of Endogenous Appearance of AA in AD}

We also reported that there might be 2 patterns that accelerate $\mathrm{AD}$ pathology, i.e. amyloid pathology [3]. One is that when ACh reaches a critical level, endogenous AA triggers a rapid cognitive decline at a moderate stage in $\mathrm{AD}$. The other begins without ACh downregulation reaching a critical level. Thus, in the second pattern, AA follows minimal ACh downregulation due to exogenous factors (i.e. medications, physical illness and mental stress]), e.g. delirium [3]. In general, when exogenous inserts are dissolved, cognitive function returns to the previous level. However, when the duration of AA exposure increases, cognitive function fails to return to baseline levels [30]. The former is related to SAA positivity at year $\mathrm{X}+3$ and the latter is related to that at year X. From this review, we confirmed that SAA is an excellent peripheral marker for AA burden in the central nervous system. 
Secondly, we reconsidered the stages of AD (MCI, mild, moderate) based on the hypothesis of endogenous appearance of AA in AD. First, we speculated that AA first appears at the moderate stage. Next, we speculated on its appearance in the MCI and mild stages. Generally speaking, $\mathrm{AD}$ pathology causes the degeneration of cholinergic neurons [23]. However, although the activity of choline acetyltransferase (ChAT, an enzyme that produces $\mathrm{ACh}$ ) is downregulated in the mild to moderate stages of AD, Gilmor et al. [31], De Kosky et al. [32] and Ikonomovic et al. [33] reported that in patients with $\mathrm{MCI}$ or early $\mathrm{AD}$, ChAT activity shows a compensatory increase so that $\mathrm{ACh}$ activity remains normal. Accordingly, our patient's ChAT activity could have been high, which could account for the fact that her ACh level was relatively normal and her cognitive function was relatively intact. This compensatory reaction to the onset of $\mathrm{AD}$ may be attributable to hyperactivity of presynaptic cholinergic neurons. If this compensatory mechanism works, then the cholinergic system is intact, rather than deteriorated. This means that when clinical symptoms occur, the cholinergic system is burdened, but still intact. Therefore, we speculate that when clinical symptoms occur, neurons are intact (i.e. not degenerated). When $\mathrm{AD}$ pathology causes clinical symptoms, such as memory disturbance, disorientations, etc., then neurons are degenerated. From these speculations, we hypothesize that in the MCI stage, AD pathology burdens the brain in AD patients, but ChAT activity is upregulated, resulting in normal ACh levels. Moreover, we also propose that in mild-stage $\mathrm{AD}, \mathrm{ACh}$ gradually decreases because hyperactivity of presynaptic neurons may cause early and rapid neuronal degeneration, consequently downregulating ChAT activity. In the moderate stage, as we mentioned, because downregulation of ACh reaches a critical level, AA appears endogenously (fig. $4 \mathrm{a}-\mathrm{c}$ ).

\section{Pharmacotherapy for AD Based on the Hypothesis of Endogenous Appearance of AA in AD}

Based on our proposed hypothesis, we can speculate on a rational pharmacotherapy for $\mathrm{AD}$. As we have previously commented, NMDA receptor hyperactivity is a key factor behind ACh downregulation, anti-inflammatory pathway downregulation, inflammatory upregulation, and inflammation-generated AA. We believe that true treatment of $\mathrm{AD}$ should start with NMDA receptor antagonists at the moderate stage, with the prescription of

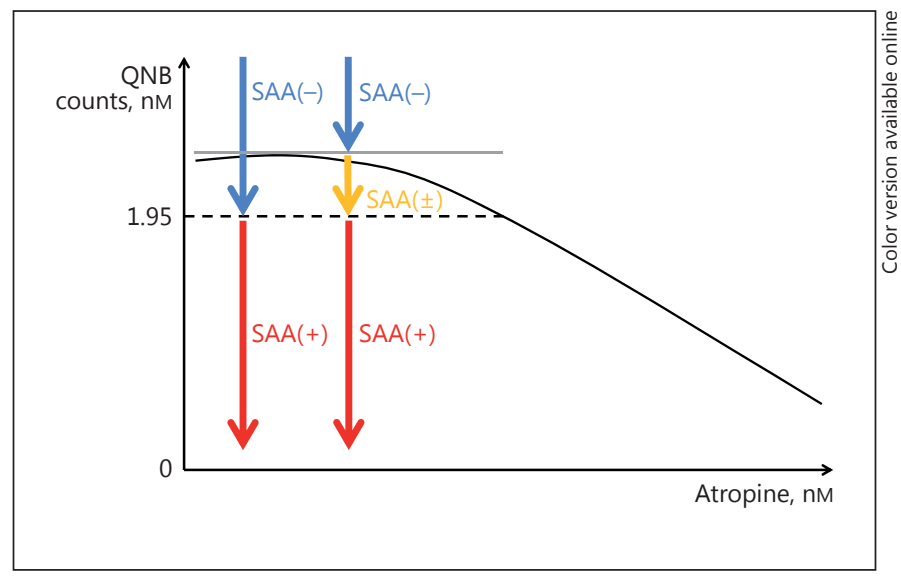

Fig. 3. The relationship between the atropine concentration in a standard solution (given in nanomolars) and the $\left[{ }^{3} \mathrm{H}\right] \mathrm{QNB}$ (titrated quinuclidinyl benzylate) counts is linear from 1.95 to $25 \mathrm{nM}$. Generally, SAA is positive if the $\left[{ }^{3} \mathrm{H}\right] \mathrm{QNB}$ counts are below the level corresponding to $1.95 \mathrm{nM}$; therefore, SAA is considered positive when the count is $\geq 1.95 \mathrm{nM}$ (left red arrow; colors refer to the online version) and undetectable when $<1.95 \mathrm{nM}$ (left blue arrow). Nevertheless, because a small amount of SAA can cause AA in the central nervous system, clinicians should keep in mind that SAA might also be positive if $\left[{ }^{3} \mathrm{H}\right] \mathrm{QNB}$ counts are above the level corresponding to $1.95 \mathrm{nM}(0 \mathrm{nM}<\mathrm{SAA}<1.95 \mathrm{nM}$; right yellow arrow $)$. 0 and $1.95 \mathrm{nM}$ are counts of the QNB, equivalent to a SAA concentration of 0 or $1.95 \mathrm{nM}$, respectively. Image courtesy of Hori et al. [2].

cholinesterase inhibitors to prevent the appearance of AA $[1,3]$.

Consequently, we advocate the careful evaluation of psychotropic therapy for $\mathrm{AD}$ patients, especially when there is evidence of AA. Prescription medication is not only a cause of worsening cognitive function (especially memory), but also of acceleration of $\mathrm{AD}$ pathology. $\mathrm{Al}$ though impairments of cognitive function can be reversed, it takes time and is typically only partial, even when the medications are discontinued [30]. It is therefore possible that long-term exposure to anticholinergic medications causes irreversible changes in $\mathrm{AD}$, and that we should avoid treating $\mathrm{AD}$ patients with medicines that cause AA. In fact, it is reported that benzodiazepines, such as anxiolytics or sedative-hypnotics, are reported to be often prescribed for elderly patients and these medications are related with a risk of $\mathrm{AD}$ [34].

$\mathrm{AD}$ is not only a mental or neuropsychiatric disorder, but also a systemic one. This is especially true during the moderate stage when hyperactivity of the inflammatory system occurs in both central and peripheral nervous tissues because of ACh downregulation and loss of inflam- 

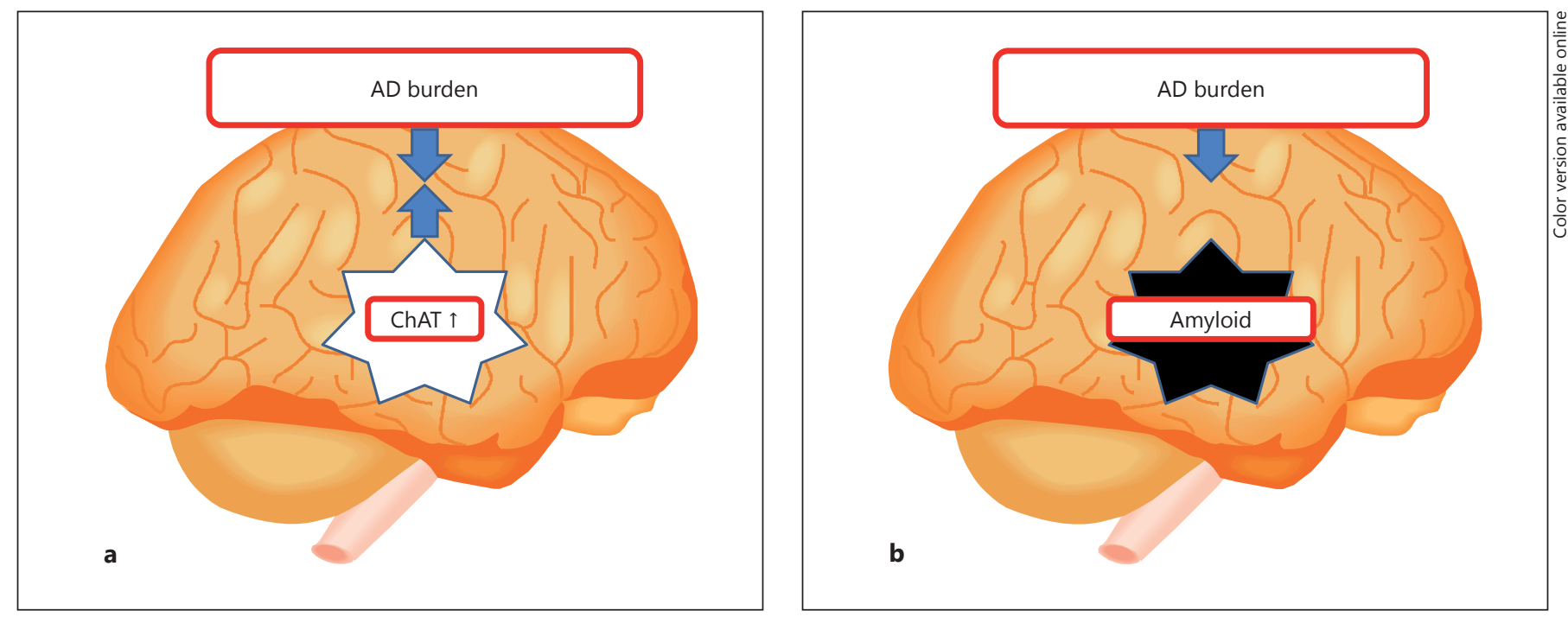

Fig. 4. a MCI stage: $\mathrm{AD}$ pathology causes a burden in the brains of patients with $\mathrm{AD}$; however, ChAT activity is upregulated and $\mathrm{ACh}$ is maintained at a normal level. b Mild stage: ACh gradually decreases because hyperactivity of presynaptic neurons may cause early and rapid neuronal degeneration with a consequent downregulation of ChAT activity. c Moderate stage: AA appears endogenously because ACh downregulation reaches a critical level.

mation control. Therefore, it is important to control peripheral illness, and to ensure that medications with AA are not prescribed [1]. In brief, we should be mindful of AA.

\section{Conclusion}

In this report, we have argued for our hypothesis of the endogenous appearance of $\mathrm{AA}$ in $\mathrm{AD}$, and have provided evidence of a patient with $\mathrm{AD}$ whose SAA became positive, possibly through endogenous mechanisms, during

long-term observation. To confirm the endogenous AA hypothesis of $\mathrm{AD}$, it is necessary to observe SAA results for greater numbers of patients with stable AD and medications over a longer time period. Our hypothesis is derived from our 2 previous reports and a review of the current literature, and consequently needs to be proven clinically. However, while we are awaiting comprehensive data in this regard, case reports, such as those presented in this paper, provide important evidence of our hypothesis. Indeed, to our knowledge, the case presentation in this article is the first to show the possibility of endogenous AA appearing in $\mathrm{AD}$ (or Lewy body disease). We 
also commented on the clinical course and staging in $\mathrm{AD}$ based on the proposed hypothesis. Finally, we considered the role of rational pharmacotherapy for $\mathrm{AD}$ based on the proposed hypothesis.

In conclusion, the hypothesis of endogenous $\mathrm{AA}$ in $\mathrm{AD}$ has 2 important clinical implications for the pharmacotherapy of AD. First, based on our endogenous AA hypothesis, AA is a biological marker for the rapid progression of AD. We should therefore regularly measure SAA in $\mathrm{AD}$ patients. The change in SAA from negative to positive probably reflects the onset of rapid disease progression, at which point NMDA receptor antagonists should be prescribed. SAA is an important biological marker of the AA burden in both the central and peripheral nervous systems; when AA appears, we should consider that the ACh system is deteriorated or at least burdened. Secondly, we should avoid medicines with AA for elderly patients, especially for $\mathrm{AD}$ patients. Both AA increases and ACh downregulation not only lead to cognitive deterioration, but also accelerate the progression of $\mathrm{AD}$ via amyloid pathology.

\section{Acknowledgments}

The funding for this study was provided by Eisai Co. Ltd., Daiichi Sankyo Inc. and Ono Pharmaceutical Co. Ltd. We thank Dr. Yutaka Takayama (Director of the Psychosomatic Medicine Memory Clinic, Federation of National Public Service Personnel Mutual Aids Associations, Tokyo Kyosai Hospital) for the suggestions regarding the amyloidogenic pattern.

\section{Disclosure Statement}

Koji Hori received lecture fees from Eisai Co. Ltd., Pfizer Japan Inc., Novartis Pharma KK, Daiichi Sankyo Inc., Ono Pharmaceutical Co. Ltd., Janssen Pharmaceutical KK, Yoshitomi Yakuhin Co. and Mitsubishi Tanabe Pharma Co. Mitsugu Hachisu received funding from Astellas Pharma Inc., Meiji Seika Pharma Co. Ltd., Dainippon Sumitomo Pharm Co. Ltd., Eli Lilly Japan KK, and Shionogi \& Co. Ltd. He also received lecture fees from Meiji Seika Pharma Co. Ltd. and Mitsubishi Tanabe Pharma Co.

\section{References}

1 Hori K, Konishi K, Akita R, Tani M, Tomioka H, Kitajima Y, Yokoyama S, Azuma K, Ikuse D, Akashi N, Yuda H, Hachisu M: Proposal of endogenous anticholinergic hypothesis in Alzheimer's disease (in Japanese). Jpn J Neuropsychopharmacol 2013;33:117-126.

$\checkmark 2$ Hori K, Konishi K, Tani M, Tomioka H, Akita R, Kitajima Y, Aoki M, Yokoyama S, Azuma K, Ikuse D, Akashi N, Hosoi M, Jinbo K, Hachisu M: Serum anticholinergic activity: a possible peripheral marker of the anticholinergic burden in the central nervous system in Alzheimer's disease. Dis Markers 2014;2014: 459013.

3 Hori K, Konishi K, Tani M, Tomioka H, Akita R, Kitajima Y, Aoki M, Kikuchi N, Ikuse D, Akashi N, Hosoi M, Jinbo K, Hachisu M: Why does the progression of Alzheimer's disease accelerate? Ann Psychiatry Ment Health 2014;2:1006.

4 Tune L, Coyle JT: Serum levels of anticholinergic drugs in treatment of acute extrapyramidal side effects. Arch Gen Psychiatry 1980;37: 293-297.

$>5$ Konishi K, Hori K, Uchida H, Watanabe K, Tominaga I, Kimura M, Hosoyamada M, Shibasaki T, Kataoka A, Hachisu M: Adverse effects of anticholinergic activity on cognitive functions in Alzheimer's disease. Psychogeriatrics 2010;10:34-38.
6 Hori K, Konishi K, Watanabe K, Uchida H, Tsuboi T, Moriyasu M, Tominaga I, Hachisu M: Influence of anticholinergic activity in serum on clinical symptoms of Alzheimer's disease. Neuropsychobiology 2011; 63:147-153.

7 Sunderland T, Tariot PN, Cohen RM, Weingartner H, Mueller EA 3rd, Murphy DL: Anticholinergic sensitivity in patients with dementia of Alzheimer type and age-matched control: a dose-response study. Arch Gen Psychiatry 1987;44:418-426.

$>8$ Thienhaus OJ, Allen A, Bennett JA, Chopra YM, Zemlan FP: Anticholinergic serum levels and cognitive performance. Eur Arch Psychiatry Clin Neurosci 1990;240:28-33.

$>9$ Chien IC, Hsu JH, Bih SH, Lin CH, Chou YJ, Lee $\mathrm{CH}$, Chou P: Prevalence, correlates, and disease patterns of antipsychotic use in Taiwan. Psychiatry Clin Neurosci 2008;62:677-684.

10 Wood-Mitchell A, James IA, Waterworth A, Swann A, Ballard C: Factors influencing the prescribing of medications by old age psychiatrists for behavioural and psychological symptoms of dementia: a qualitative study. Age Ageing 2008;37:547-552.

11 Perry EK, Kilford L, Lees AJ, Burn DJ, Perry $\mathrm{RH}$ : Increased Alzheimer pathology in Parkinson's disease related to antimuscarinic drugs. Ann Neurol 2003;54:235-8.

$\checkmark 12$ Lu CJ, Tune LE: Chronic exposure to anticholinergic medications adversely affects the course of Alzheimer disease Am J Geriatr Psychiatry $2003 ; 11: 458-461$.
13 Tune L, Carr S, Hoag E, Cooper T: Anti-cholinergic effects of drugs commonly prescribed for the elderly: potential means for assessing risk of delirium. Am J Psychiatry 1992;149: 1393-1394.

14 Flacker JM, Lipsitz LA: Serum anticholinergic activity changes with acute illness in elderly medical patients. J Gerontol A Biol Sci Med Sci 1999;54:M12-M16.

15 Plaschke K, Kopitz J, Mattern J, Martin E, Teschendorf P: Increased cortisol levels and anticholinergic activity in cognitively unimpaired patients. J Neuropsychiatry Clin Neurosci 2010;22:433-441.

-16 Hori K, Konishi K, Tomioka H, Tani M, Minegishi G, Tanaka M, Akita R, Yokoyama S, Oshio T, Hachisu M: Serum anticholinergic activity: a biomarker for rapid progression of Alzheimer's disease. J Autacoids 2012, DOI: org/10.4172/2161-0479.S4-001.

17 Horiuchi Y, Fujii T, Kamimura Y, Kawashima $\mathrm{K}$ : The endogenous, immunologically active peptide apelin inhibits lymphocytic cholinergic activity during immunological responses. J Neuroimmunol 2003;144:46-52.

18 Nazarov PG, Krylova IB, Evdokimova NR, Nezhinskaya GI, Butyugov AA: C-reactive protein: a pentraxin with anti-acetylcholine activity. Life Sci 2007;80:2337-2341. 
19 Pavlov VA, Ochani M, Gallowitsch-Puerta M, Ochani K, Huston JM, Czura CJ, Al-Abed Y, Tracey KJ: Central muscarinic cholinergic regulation of the systemic inflammatory response during endotoxemia. Proc Natl Acad Sci USA 2006;103:5219-5223.

20 Mabley JG, Pacher P, Szabo C: Activation of the cholinergic anti-inflammatory pathway reduces ricin-induced mortality and organ failure in mice. Mol Med 2009;15:166-172.

-21 Vandame D, Ulmann L, Teigell M, Prieto-Cappellini M, Vignon J, Privat A, PerezPolo R, Nesic O, Hirbec H: Development of NMDAR antagonists with reduced neurotoxic side effects: a study on GK11. PLoS One 2013;8:e81004.

22 Gahring LC, Meyer EL, Rogers SW: Nicotineinduced neuroprotection against $\mathrm{N}$-methyl-D-aspartic acid or beta-amyloid peptide occur through independent mechanisms distinguished by pro-inflammatory cytokines. J Neurochem 2003;87:1125-1136.

23 Whitehouse PJ, Price DL, Struble RG, Clark AW, Coyle JT, Delon MR: Alzheimer's disease and senile dementia: loss of neurons in the basal forebrain. Science 1982;215:12371239.

24 Hori K, Konishi K, Tomioka H, Minegishi G, Tani M, Tanaka H, Akita R, Yokoyama S, Oshio T, Hachisu M: Memantine abolishes anticholinergic activity in patient with Alzheimer's disease at moderate stage. J Alzheimers Dis Parkinsonism 2012;2:3.
5 Folstein MF, Folstein SE, McHugh PR: 'Minimental state': a practical method for grading the cognitive state of patients for the clinician. J Psychiatr Res 1975;12:189-198.

26 Morris JC, Cummings J: Mild cognitive impairment (MCI) represents early-stage $\mathrm{Alz}$ heimer's disease. J Alzheimers Dis 2005;7: 235-239.

27 McKhann G, Drachman D, Folstein M, Katzman R, Price D, Stadlan EM: Clinical diagnosis of Alzheimer's disease: report of the NINCDS-ADRDA Work Group under the auspices of Department of Health and $\mathrm{Hu}$ man Services Task Force on Alzheimer's Disease. Neurology 1984;34:939-944.

-28 Konishi K, Hori K, Tomioka H, Minegishi G, Tani M, Tanaka H, Akita R, Yokoyama S, Oshio T, Hachisu M: Donepezil abolishes anticholinergic activity in a patient with amnesia. Pharmacology 2013;91:86-91.

29 Hori K, Funaba Y, Konishi K, Moriyasu M, Hirata K, Oyamada R, Tominaga I, Inada T: Assessment of pharmacological toxicity using serum anticholinergic activity in a patient with dementia. Psychiatry Clin Neurosci 2005;59:508-510.
30 Tönne U, Hiltunen AJ, Vikander B, Engelbrektsson K, Bergman H, Bergman I, Leifman $\mathrm{H}$, Borg S: Neuropsychological changes during steady-state drug use, withdrawal and abstinence in primary benzodiazepine-dependent patients. Acta Psychiatr Scand 1995;91: 299-304.

- 31 Gilmor ML, Erickson JD, Varoqui H, Hersh LB, Bennett DA, Cochran EJ, Mufson EJ, Levey AI: Preservation of nucleus basalis neurons containing choline acetyltransferase and the vesicular acetylcholine transporter in the elderly with mild cognitive impairment and early Alzheimer's disease. J Comp Neurol 1999;411:693-704.

32 De Kosky ST, Ikonomovic MD, Styren SD, Beckett L, Wisniewski S, Bennett DA, Cochran EJ, Kordower JH, Mufson EJ: Upregulation of choline acetyltransferase activity in hippocampus and frontal cortex of elderly subjects with mild cognitive impairment. Ann Neurol 2002;51:145-155.

33 Ikonomovic MD, Mufson EJ, Wuu J, Bennett DA, De Kosky ST: Reduction of choline acetyltransferase activity in primary visual cortex in mild to moderate Alzheimer's disease. Arch Neurol 2005;62:425-430.

- 34 Billioti de Gage S, Moride Y, Ducruet T, Kurth T, Verdoux H, Tournier M, Pariente A, Bégaud B: Benzodiazepine use and risk of Alzheimer's disease: case-control study. BMJ 2014;349:g5205. 